\title{
Given the problem of projection, are heat maps an oxymoron?
}

\author{
Daniel "daan" Strebe a \\ ${ }^{a}$ Tableau Software,dstrebe@tableau.com
}

Keywords: map projection, heat map, data visualization

\begin{abstract}
:
With the proliferation of data analysis and visualization tools, we see more and more heat maps. But should we? Are such displays meaningful? At large scales, heat maps need not be controversial (though common tools can blight even simple cases). But what about small-scale maps? Is anyone thinking about the effects of projection on heat maps? How do map projections change the semantics of heat maps? What projections permit meaningful heat maps? How should heat maps be calculated in the presence of a map projection? We explore these problems and questions in this presentation to offer critique and advice.
\end{abstract}

For the purposes of this discussion, a heat map is a representation of the density or magnitude of a spatial phenomenon on two dimensions, treating the density or magnitude as a continuous measure whether or not the underlying phenomenon is continuous. If the data are too sparse in the presentation space, then the fiction of continuity ought to be avoided; a heat map would not be an appropriate visualization. While real world examples of heat maps that violate this principle are easy to find, we take the principle for granted and do not elaborate further here.

Unfortunately, there are several other ways to construct ineffective heat maps. One of the primary offenses is to ignore the effect of map projection on the presentation of density. It should be clear that a projection whose area measure varies widely across the presentation space necessarily distorts density. If the heat map is a presentation of density - which most are - then poor choice of projection would contradict the purpose of a heat map. The result would be a blatant fiction.

Surprisingly, the Mercator projection often can be found in small scale heat maps, for the reason that the projection is common, is the default in many sets of tooling, and is sometimes the only projection available with the set of map construction tools. And yet, as far as density variation goes, a worse case than Mercator cannot be found among common projections.

Even if density remains constant across the map, a poor heat map could be generated if the analysis for the heat map mixes phenomenon space, which is geographic, with projected space, which is not. Common tools commit this fallacy. The result is that a phenomenon whose density diminishes radially (for example) from a hot point might show as concentric circles of decreasing intensity on the projected map, whereas we would expect elongations of the heat field in accordance with the projection's distortion metric.

We conclude that, while it is possible to construct responsible heat maps of geographic data, there are several pitfalls. Among these pitfalls, we find that common tools conspire to assist in the presentation of fiction instead of fact. 


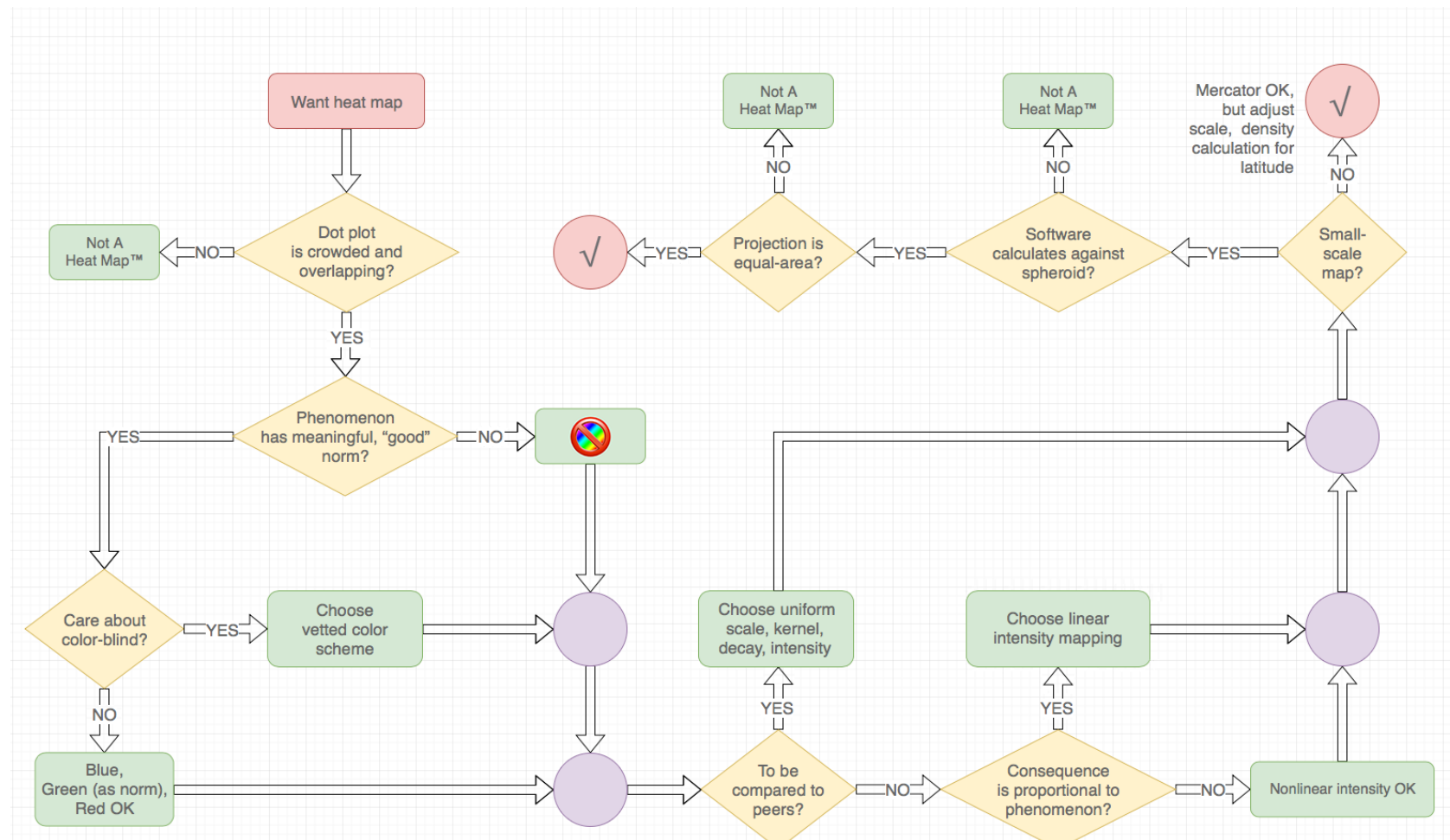

Figure 1. Are my data and presentation options appropriate for a heat map? 